\title{
Seletividade de Acaricidas a Insetos e Ácaros Predadores em Citros
}

\author{
Antonio C. dos Santos ${ }^{1}$ e Santin Gravena ${ }^{2}$ \\ ${ }^{1}$ Departamento de Entomologia e Nematologia, FCAV/UNESP, \\ 14870.000, Jaboticabal, SP.
}

${ }^{2}$ Gravena Manejo Ecológico de Pragas Ltda. Rua Monteiro Lobato, 856, 14870.000, Jaboticabal, SP.

An. Soc. Entomol. Brasil 26(1): 99-105 (1997)

Selectivity of Acaricides to Predatory Insects and Mites in Citrus Orchards

\begin{abstract}
The selectivity of acaricides to predatory insects and mites was studied in two citrus orchards in São Paulo State in 1993 and 1994. Acaricides used (g.a.i /1001) were: in 1993, pyridaben 20 EC, $20 \mathrm{WP}$ and $75 \mathrm{WP}$ (10 and 15), fenbutatin oxide (40) and control; in 1994 pyridaben 20 EC and 75 WP (10 and 20), fenbutatin oxide (40), cyhexatin (25) and control. Evaluations were conducted up to 21 days after spraying. Adults and larvae of Pentilia egena Muls. and Coccidophilus citricola Bréthes, adults of Cycloneda sanguinea L., Azya sp. and Calloeneis sp. and larvae ofchrysopids were counted on the canopy of the tree. Euseius sp. and Iphiseiodes zuluagai Denmark \& Muma were counted on 10 leaves from the canopy. The products were classified as: harmless $(<25 \%$ of mortality); slightly harmful (25 to $50 \%)$; moderately harmful (51 to $75 \%$ ), and harmful (>75\%). All products were harmless to adults of $P$. egena; fenbutatin caused $<25 \%$ of mortality, pyridaben killed 25 to $50 \%$ and cyhexatin 51 to $75 \%$ of $P$. egena larvae. Pyridaben 20 EC and 75 WP were slightly and moderately harmful, cyhexatin caused $>75 \%$ of mortality and fenbutatin was harmless to adults of $C$. citricola. Concerning C. citricola, fenbutatin killed 25 to $50 \%$ of the larvae, pyridaben $20 \mathrm{EC}$ and $75 \mathrm{WP}$ caused 51 to $75 \%$ mortality while cyhexatin caused $>75 \%$ mortality. All treatments killed more than $75 \%$ of the phytoseiids up to 21 days except fenbutatin oxide which was harmful up to 14 days.
\end{abstract}

KEY WORDS: Insecta, Acari, Coccinellidae, Phytoseiidae, pesticides.

RESUMO - Estudou-se a seletividade de acaricidas a insetos e ácaros predadores em pomares citrícolas localizados em Monte Alto e Vista Alegre do Alto, SP, em 1993 e 1994. Os acaricidas nas dosagens testadas (em g.i.a/1001) foram: em 1993, pyridaben 20 CE, 20 PM e 75 PM (10 e 15), óxido fenbutatin (40) e testemunha; em 1994, pyridaben 20 CE e pyridaben 75 PM (10 e 20), óxido fenbutatin (40), cyhexatin (25) e testemunha. Larvas e adultos de Pentilia egena Muls. e Coccidophilus citricola Bréthes, adultos de Cycloneda sanguinea L., Azya sp. e Calloeneis sp. e larvas de crisopídeos foram observados nas plantas, previamente e a 1, 3, 7, 10, 14 e 21 dias após a aplicação. Para contagem de Euseius sp. e Iphiseiodes zuluagai Denmark \& Muma, 10 folhas do interior da planta foram coletadas e observadas. Os produtos foram classificados em: seletivo 
(até $25 \%$ de mortalidade); levemente tóxico (25 a 50\%; moderadamente tóxico (51 a $75 \%$ ) e tóxico (> 75\% de mortalidade). Os resultados indicaram que todos os produtos foram seletivos a adultos de $P$. egena, porém, pyridaben causou de 25 a $50 \%$ e cyhexatin de 51 a $75 \%$ de mortalidade de larvas. Pyridaben 20 CE e 75 PM reduziram em 25 a $50 \%$ e 51 a $75 \%$ respectivamente o número de adultos de C. citricola; já cyhexatin foi tóxico e fenbutatin seletivo a adultos desta espécie. Em relação a larvas de C. citricola mortalidade de 51 a $75 \%$ foi causada por pyridaben $20 \mathrm{CE}$ e $75 \mathrm{PM}$, maior que $75 \%$ por cyhexatin e entre 25 e $50 \%$ no caso de fenbutatin. Todos os acaricidas foram tóxicos aos ácaros predadores por mais de 21 dias, no entanto, fenbutatin apresentou efeito residual menor (14 dias).

PALAVRAS-CHAVE: Insecta, Acari, Coccineilidae, Phytoseiidae, pesticidas.

O uso exclusivo de agrotóxicos de largo espectro de ação, embora tenha o objetivo de maximizar a produção mediante o aumento de eficiência leva a efeitos colaterais indesejáveis e inevitáveis (Hoyt \& Buits 1974). Os inseticidas-acaricidas e acaricidas específicos são largamente empregados em pulverizações contra os ácaros Phyllocoptruta oleivora Ashmead e Brevipalpus phoenicis Geijskes (Gravena 1984). Os primeiros, necessitam de estudos mais apurados por serem, de maneira geral, tóxicos aos inimigos naturais, no entanto, os acaricidas específicos são indicados por serem seletivos fisiológicos aos predadores, isto é, tóxicos somente aos organismos alvo (Hoyt \& Buris 1974). Pyridaben é um acaricida com potencial de utilização para o controle de B. phoenicis em citros (Moraes et al. 1995). Quanto a sua seletividade. Santos (1995), verificou que pyridaben na formulação PM mostrou maior toxicidade que a formulação $\mathrm{CE}$ a Coccidophilus citricola Bréthes, sendo porém o produto considerado como seletivo. Desta forma, o objetivo do trabalho foi testar a seletividade de pyridaben a insetos e ácaros predadores comuns em pomares de citros.

\section{Material e Métodos}

Os estudos foram conduzidos em pomares de laranja cv. Pêra Rio em Monte Alto (dez anos de idade e Vista Alegre do Alto, SP (com quatro anos). A aplicação dos acaricidas ocorreu em 21/01/1993 no ensaio I sendo utilizados os seguintes tratamentos e dosagens em g.i.a/1001: pyridaben $20 \mathrm{CE}$ (10 e 15); pyridaben20 PM (10 e 15); pyridaben 75 PM (10 e 15); óxido fenbutatin (40) e testemunha. No ensaio II a aplicação foi realizada em 22/02/ 1994 utilizando-se os seguintes tratamentos e dosagens em g.i.a/1001: pyridaben $20 \mathrm{CE}$ (10 e 20); pyridaben 75 PM (10 e 20): óxido fenbutatin (40); cyhexatin (25) e testemuna. Em ambos os ensaios, a pulverização foi feita através de pulverizador costal motorizado sendo a calda aplicada até o ponto de escorrimento (Matuo 1990). O delineamento foi o de blocos ao acaso com quatro repetições sendo cada uma constituída de uma planta. As avaliações dos predadores foram realizadas a $0,1,3,10$ e 17 dias após a aplicação (DAA) no ensaio I e a $0,1,3,7,14$ e 21 DAA no ensaio II caminhando-se ao redor da planta e anotando-se o número de larvas e adultos de coccinelídeos como Pentilia egena Muls. e C. citricola Bréthes, adultos de Cycloneda sanguínea L., Azya sp., Calloeneis sp. e larvas de crisopídeos. No ensaio II, em cada parcela foram coletadas 10 folhas do interior da planta para contagem visual de ácaros fítoseídeos (Euseius sp. e Iphiseiodes zuluagai Denmark \& Muma) (Santos \& Gravena 1995). Os dados referentes ao número de adultos e larvas dos 
predadores/planta e número de fitoseídeos/ 10 folhas foram transformados em $\log (x+1)$ e submetidos ao teste de Tukey. As percentagens de redução foram calculadas segundo a fórmula proposta por Henderson \& Tilton (1955). Para a classificação dos produtos quanto a sua toxicidade foi utilizada a tabela de Hassan (1985) para ensaios de campo que agrupa os produtos em quatro classes: não tóxico ou seletivo (até $25 \%$ de mortalidade); levemente tóxico (25a 50\%); moderadamente tóxico (51 a $75 \%$ ) e 4) tóxico (> $75 \%$ de mortalidade).

\section{Resultados e Discussão}

Em 1993 a população de adultos de $P$. egena foi baixa (2 a 4 indivíduos/planta) porém homogênea (Tabela 1). Em nenhuma data, nenhum produto, dosagem ou formulação diferiu significativamente da testemunha. Observou-se que a 1 DAA pyridaben 75 PM a 10 e 15 g.i.a/ 1001 reduziu em 40 e $47 \%$ o número de adultos de $P$. egena. Essa percentagem de redução diminui ao longo das avaliações sendo que a partir dos 10 DAA nenhum efeito dos produtos foi verificado mostrando um baixo efeito residual dos mesmos. Óxido fenbutatin destacou-se por não apresentar qualquer redução $(0 \%)$ em todas as datas. No ensaio II (1994), previamente à aplicação o número de joaninhas era de 14 a 23 adultos/ planta (Tabela 1). A 1 DAA não se constatou diferença estatística entre os tratamentos, no entanto foi observada uma redução do coccinelídeo nas parcelas tratadas com pyridaben 75 PM a 20 g.i.a/1001 (25\%) e cyhexatin (57\%),

Tabela 1. Número médio e percentagem de redução de adultos e larvas de Pentilia egena após a aplicação de acaricidas em plantas cítricas. 1993/94.

\begin{tabular}{|c|c|c|c|c|c|c|}
\hline \multirow{2}{*}{$\begin{array}{l}\text { Tratamentos } \\
\text { (g.i.a/100 1) }\end{array}$} & \multicolumn{6}{|c|}{ Dias após a aplicação } \\
\hline & \multicolumn{3}{|c|}{$\mathrm{N}^{\circ}$ adultos/planta (\% redução) } & \multicolumn{3}{|c|}{$\mathrm{N}^{\circ}$ larvas/planta (\% redução) } \\
\hline 1993 & 0 & 1 & 10 & 0 & 1 & 10 \\
\hline Pyridaben 20CE (10) & $3,3 \mathrm{a}$ & $2,0 \mathrm{a}(17)$ & $1,7 \mathrm{a}(0)$ & $2,7 \mathrm{a}$ & $1,5 \mathrm{a}(58)$ & $10,6 \mathrm{a}(0)$ \\
\hline Pyridaben 20PM (10) & $2,0 \mathrm{a}$ & $1,3 \mathrm{a}(11)$ & $3,6 \mathrm{a}(0)$ & $2,0 \mathrm{a}$ & $1,3 \mathrm{a}(52)$ & $5,0 \mathrm{a}(30)$ \\
\hline Pyridaben 75PM (10) & $3,0 \mathrm{a}$ & $1,3 \mathrm{a}(40)$ & $4,3 \mathrm{a}(0)$ & $3,0 \mathrm{a}$ & $3,0 \mathrm{a}(26)$ & $8,0 \mathrm{a}(25)$ \\
\hline Pyridaben 20CE (15) & $4,0 \mathrm{a}$ & $2,3 a(21)$ & $2,7 \mathrm{a}(0)$ & $1,7 \mathrm{a}$ & $1,3 \mathrm{a}(43)$ & $4,3 a(29)$ \\
\hline Pyridaben 20PM (15) & $3,7 \mathrm{a}$ & $1,7 \mathrm{a}(37)$ & $3,0 \mathrm{a}(0)$ & $2,7 \mathrm{a}$ & $2,0 \mathrm{a}(45)$ & $8,3 a(13)$ \\
\hline Pyridaben 75PM (15) & $3,3 \mathrm{a}$ & $1,3 \mathrm{a}(47)$ & $4,3 \mathrm{a}(0)$ & $2,0 \mathrm{a}$ & $1,5 \mathrm{a}(44)$ & $5,3 a(25)$ \\
\hline Óxido Fenbutatin (40) & $2,3 \mathrm{a}$ & $2,0 \mathrm{a}(0)$ & $2,0 \mathrm{a}(0)$ & $2,3 a$ & $2,3 \mathrm{a}(26)$ & $6,3 \mathrm{a}(23)$ \\
\hline Testemunha & $3,7 \mathrm{a}$ & $2,7 \mathrm{a}$ & $2,0 \mathrm{a}$ & $1,7 \mathrm{a}$ & $2,3 \mathrm{a}$ & $6,0 \mathrm{a}$ \\
\hline C.V. & 38,4 & 37,9 & 33,7 & 40,2 & 71,5 & 33,5 \\
\hline 1994 & 0 & 1 & 14 & 0 & 1 & 14 \\
\hline Pyridaben 20CE (10) & $17,5 \mathrm{a}$ & $22,8 \mathrm{a}(0)$ & $18,0 \mathrm{a}(0)$ & $50,3 \mathrm{a}$ & $46,7 \mathrm{a}(40)$ & $3,2 \mathrm{a}(56)$ \\
\hline Pyridaben 75PM (10) & $16,5 \mathrm{a}$ & $21,3 \mathrm{a}(0)$ & $20,0 \mathrm{a}(0)$ & $78,7 \mathrm{a}$ & $90,0 \mathrm{a}(25)$ & $9,0 \mathrm{a}(21)$ \\
\hline Pyridaben 20CE (20) & $14,0 \mathrm{a}$ & $25,8 \mathrm{a}(0)$ & $14,8 \mathrm{a}(0)$ & $64,0 \mathrm{a}$ & $53,2 \mathrm{a}(45)$ & $3,2 \mathrm{a}(65)$ \\
\hline Pyridaben 75PM (20) & $15,7 \mathrm{a}$ & $13,0 \mathrm{a}(25)$ & $19,0 \mathrm{a}(0)$ & 76,0 a & $55,0 \mathrm{a}(52)$ & $6,8 \mathrm{a}(39)$ \\
\hline Óxido Fenbutatin (40) & $21,0 \mathrm{a}$ & $1,5 \mathrm{a}(6)$ & $22,0 \mathrm{a}(0)$ & $75,7 \mathrm{a}$ & $89,8 \mathrm{a}(22)$ & 6,8 a (39) \\
\hline Cyhexatin (25) & $15,0 \mathrm{a}$ & 7,0a (57) & $16,5 \mathrm{a}(0)$ & 34,2 a & $23,8 \mathrm{a}(55)$ & 3,3a (34) \\
\hline Testemunha & $23,2 \mathrm{a}$ & $25,5 \mathrm{a}$ & $13,8 \mathrm{a}$ & $43,0 \mathrm{a}$ & $66,5 \mathrm{a}$ & $6,3 \mathrm{a}$ \\
\hline C.V. & 29,5 & 23,1 & 18,0 & 23,0 & 20,2 & 55,7 \\
\hline
\end{tabular}


ocorrendo porém um aumento no número de indivíduos nesses tratamentos a partir de 3 DAA, não se observando efeito de redução por parte dos produtos. Em relação a cyhexatin e óxido fenbutatin, tais resultados são semelhantes aos obtidos por Morse et al. (1987) sobre Cryptolaemus montrouzieri Muls.

No que se refere a larvas de P. egena (Tabela 1), no ensaio I (1993), a população foi muito baixa (1,7 a 3 indivíduos/planta) por ocasião da avaliação prévia não sendo detectada diferença estatística em nenhuma das datas. Entretanto a 1 DAA todos os produtos reduziram o número de larvas/planta (entre 43 e $58 \%$ ), sendo óxido fenbutatin e pyridaben 75 PM a 10 g.i.a/100 1 os tratamentos que apresentaram menor percentagem de redução (26\%).
Aos 10 DAA verificou-se um aumento significativo no número de larvas/planta (4 a 10) em todos os tratamentos. Isto mostra não haver efeito dos produtos sobre ovos deste coccinelídeo que, por serem colocados sob as carapaças de cochonilhas, ficam protegidos do contato com agrotóxicos. No ensaio II (1994) a população ocorreu em níveis mais elevados (34 a 78 indivíduos/planta na avaliação prévia) e de maneira homogênea (Tabela 1). A 1 DAA apesar de não diferirem significativamente da testemunha verificou-se uma redução de larvas, entre $40 \%$ e $55 \%$ em quase todos os tratamentos, exceção a pyridaben 75 PM a 10 g.i.a/1001 (25\%) e óxido fenbutatin (22\%). A partir da avaliação de 1 DAA a população decresceu em todos os tratamentos caindo a

Tabela 2. Número médio e percentagem de redução de adultos e larvas de Coccidophilus citricola e $\mathrm{n}^{\circ}$ de adultos de Cycloneda sanguinea, Calloeneis sp. e Azya sp. após a aplicação de acaricidas em plantas cítricas. 1994.

\begin{tabular}{|c|c|c|c|c|c|c|c|c|c|}
\hline \multirow{3}{*}{$\begin{array}{l}\text { Tratamentos } \\
\text { (g.i.a/100 1) }\end{array}$} & \multicolumn{9}{|c|}{ Dias após a aplicação } \\
\hline & \multicolumn{3}{|c|}{$\begin{array}{l}\mathrm{N}^{\circ} \text { adultos de } \\
\text { C. citricola/planta } \\
\text { (\% redução) }\end{array}$} & \multicolumn{5}{|c|}{$\begin{array}{c}\mathrm{N}^{\circ} \text { larvas de } \\
\text { C. citricola/planta } \\
(\% \text { redução })\end{array}$} & $\begin{array}{l}\mathrm{N}^{\circ} \text { total de adultos } \\
\text { de C. sanguinea, } \\
\text { Calloeneis sp. e } \\
\text { Azya sp./planta }\end{array}$ \\
\hline & 0 & 1 & 7 & 21 & 0 & 1 & 7 & 21 & 21 \\
\hline Pyridaben 20CE (10) & $9,0 \mathrm{a}$ & $4,0 \mathrm{a}(58)$ & $5,5 \mathrm{a}(51)$ & $1,0 \mathrm{a}(0)$ & $3,0 \mathrm{a}$ & $2,0 \mathrm{ab}(57)$ & $0,5 \mathrm{a}(0)$ & 0 & 1,0 a 1,3 a $1,0 a$ \\
\hline Pyridaben 75PM (10) & $16,7 \mathrm{a}$ & $3,5 \mathrm{ab}(80)$ & $4,8 \mathrm{a}(77)$ & $1,8 \mathrm{a}(0)$ & $5,7 \mathrm{a}$ & $3,8 \mathrm{ab}(57)$ & $1,0 \mathrm{a}(0)$ & 0 & 1,5 a 1,0 a 0,8 a \\
\hline Pyridaben 20CE (20) & $9,0 \mathrm{a}$ & $6,0 \mathrm{a}(37)$ & $6,8 \mathrm{a}(39)$ & $1,0 \mathrm{a}(0)$ & $8,5 \mathrm{a}$ & $3,0 \mathrm{ab}(77)$ & $0,8 \mathrm{a}(11)$ & 0 & 2,0 a 0,8 a $1,3 a$ \\
\hline Pyridaben 75PM (20) & $8,2 \mathrm{a}$ & $2,5 \mathrm{ab}(71)$ & $5,5 \mathrm{a}(46)$ & $1,5 \mathrm{a}(0)$ & $6,5 \mathrm{a}$ & $3,5 \mathrm{ab}(65)$ & $0,3 a(56)$ & 0 & 0,8 a 1,3 a $0,5 a$ \\
\hline Oxido Fenbutatin (40) & $11,2 \mathrm{a}$ & 10,5 a (16) & $10,8 \mathrm{a}(23)$ & $1,3 \mathrm{a}(0)$ & $7,0 \mathrm{a}$ & $8,0 \mathrm{~b}(27)$ & $0,3 a(59)$ & 0 & 2,0 a 0,8 a $0,8 \mathrm{a}$ \\
\hline Cyhexatin (25) & $7,5 \mathrm{a}$ & $0,3 b(96)$ & $3,5 \mathrm{a}(63)$ & $0,5 \mathrm{a}(0)$ & $3,0 \mathrm{a}$ & $0,3 \mathrm{a}(94)$ & $0,0 \mathrm{a}(100)$ & 0 & 0,5 a 1,0 a $1,0 a$ \\
\hline Testemunha & $12,0 \mathrm{a}$ & $12,8 \mathrm{a}$ & $15,0 \mathrm{a}$ & $1,3 \mathrm{a}$ & $4,7 \mathrm{a}$ & $7,3 \mathrm{ab}$ & $0,5 \mathrm{a}$ & 0 & 1,5 a 1,8 a $1,3 \mathrm{a}$ \\
\hline C.V. & 25,40 & 40,06 & 54,79 & 99,65 & 38,70 & 55,12 & 98,0 & - & $-74,43 \quad 88,25 \quad 86,59$ \\
\hline
\end{tabular}

níveis baixos (3 a 9 larvas / planta) até os 14 DAA. No decorrer do ensaio foi verificado um aumento de adultos de P. egena, ou seja, a redução provavelmente de larvas não ocorreu por efeito dos produtos e sim pelo empupamento das mesmas, $\mathrm{O}$ aumento no número de adultos comprova a seletividade dos pro- dutos sobre pupas e larvas de último instar do predador .

Em relação a C. citricola (Tabela 2) a 1 DAA notou-se uma redução de adultos da joaninha, principalmente por parte de pyridaben 75 PM a 10 e 15 g.i.a/100 $1(80 \%$ e $71 \%$ respectivamente) e cyhexatin $(96 \%)$. Óxido 
Tabela 3. Número médio e percentagem de redução ácaros predadores (Iphiseiodes zuluagai e Euseius sp.) e crisopídeos após a aplicação de acaricidas em plantas cítricas. 1993/94.

\begin{tabular}{|c|c|c|c|c|c|c|c|}
\hline $\begin{array}{l}\text { Tratamentos } \\
(\text { g.i.a/100 1) }\end{array}$ & $\begin{array}{r}\text { Dias ap } \\
N^{\circ} \mathrm{d} \\
\text { crisop }\end{array}$ & $\begin{array}{l}\text { s a apl } \\
\text { larvas } \\
\text { deos/pl }\end{array}$ & $\begin{array}{l}\text { cação } \\
\text { de } \\
\text { anta }\end{array}$ & & $\begin{array}{l}\text { Dias apó } \\
\mathrm{N}^{\circ} \text { de } \\
10 \text { folhas }\end{array}$ & $\begin{array}{l}\text { a aplicaç } \\
\text { toseídeos/ } \\
\text { \% reduçã }\end{array}$ & \\
\hline 1993 & 0 & 1 & 17 & 0 & 1 & 14 & 21 \\
\hline Pyridaben 20CE (10) & $0,3 \mathrm{a}$ & 0,7 a & $0,3 \mathrm{a}$ & - & - & - & - \\
\hline Pyridaben 20PM (10) & $0,3 \mathrm{a}$ & $0,7 \mathrm{a}$ & $0,7 \mathrm{a}$ & - & - & & \\
\hline Pyridaben 75PM (10) & $0,7 \mathrm{a}$ & $0,3 \mathrm{a}$ & $0,3 \mathrm{a}$ & - & - & - & - \\
\hline Pyridaben 20CE (15) & $0,3 \mathrm{a}$ & $0,3 \mathrm{a}$ & $0,3 \mathrm{a}$ & - & - & - & - \\
\hline Pyridaben 20PM(15) & $0,7 \mathrm{a}$ & $1,3 \mathrm{a}$ & $0,3 \mathrm{a}$ & - & - & - & - \\
\hline Pyridaben 75PM (15) & $0,3 \mathrm{a}$ & $0,7 \mathrm{a}$ & $1,0 \mathrm{a}$ & - & - & - & - \\
\hline Óxido Fenbutatin (40) & $0,7 \mathrm{a}$ & $0,0 \mathrm{a}$ & $0,3 \mathrm{a}$ & - & - & - & - \\
\hline Testemunha & $2,0 \mathrm{a}$ & $2,0 \mathrm{a}$ & $0,3 \mathrm{a}$ & - & - & - & - \\
\hline C.V. & 160,06 & 97,98 & 117,83 & & & & \\
\hline 1994 & 0 & 1 & 14 & 0 & 1 & 14 & 21 \\
\hline Pyridaben 20CE (10) & $0,8 \mathrm{a}$ & $0,3 \mathrm{a}$ & $0,5 \mathrm{ab}$ & $2,5 \mathrm{a}$ & $0,3 \mathrm{a}(90)$ & $0,0 b(100)$ & $0,0 \mathrm{~b}(100)$ \\
\hline Pyridaben 75PM (10) & $0,8 \mathrm{a}$ & $1,0 \mathrm{a}$ & $2,0 \mathrm{a}$ & $4,5 \mathrm{a}$ & $0,5 \mathrm{a}(91)$ & $0,3 b(96)$ & $0,5 \mathrm{~b}(93)$ \\
\hline Pyridaben 20CE (20) & $0,5 \mathrm{a}$ & $0,5 \mathrm{a}$ & $1,0 \mathrm{ab}$ & $3,5 \mathrm{a}$ & $0,5 \mathrm{a}(89)$ & $0,0 b(100)$ & $0,0 \mathrm{~b}(100)$ \\
\hline Pyridaben 75PM (20) & $0,5 \mathrm{a}$ & $0,8 \mathrm{a}$ & $0,5 \mathrm{ab}$ & $5,7 \mathrm{a}$ & $1,0 \mathrm{a}(86)$ & $0,3 \mathrm{~b}(97)$ & $0,3 b(97)$ \\
\hline Oxido Fenbutatin (40) & $1,5 \mathrm{a}$ & $0,3 \mathrm{a}$ & $0,3 \mathrm{~b}$ & $2,8 \mathrm{a}$ & $0,8 \mathrm{a}(76)$ & $0,8 \mathrm{~b}(82)$ & $2,2 \mathrm{ab}(54)$ \\
\hline Cyhexatin (25) & $0,5 \mathrm{a}$ & $1,8 \mathrm{a}$ & $0,3 \mathrm{~b}$ & 3,8 a & $1,5 \mathrm{a}(67)$ & $0,8 \mathrm{~b}(87)$ & $1,0 \mathrm{ab}(84)$ \\
\hline Testemunha & $0,8 \mathrm{a}$ & $0,5 \mathrm{a}$ & $0,3 b$ & $2,3 \mathrm{a}$ & $2,8 \mathrm{a}$ & $3,7 \mathrm{a}$ & $4,0 \mathrm{a}$ \\
\hline C.V. & 106,10 & 107,23 & 92,38 & 25,94 & 107,86 & 72,61 & 84,98 \\
\hline
\end{tabular}

fenbutatin reduziu o número de adultos em $12 \%$ e pyridaben $20 \mathrm{CE}$ a 10 a 15 g.i.a/1001, em $37 \%$ e $58 \%$ respectivamente, apresentandose como moderadamente tóxico. Verificou-se uma maior toxicidade de pyridaben na formulação $\mathrm{PM}$ em relação a $\mathrm{CE}$, concordando com os resultados de Santos (1995). Entre 1 e 7 DAA a população de adultos aumentou para então decrescer até os 21 DAA em todos os tratamentos. Esse aumento demonstra que os acaricidas exercem apenas efeito de choque sobre $C$. citricola, sendo óxido fenbutatin o mais seletivo (23\% de redução) (Tabela 2 ). Quanto às larvas (Tabela 2), observou-se que cyhexatin causou uma redução acentuada
(94\% e $100 \%$ a 1 e 7 DAA). Pyridaben não diferiu entre as dosagens e formulações (57\% a $67 \%$ de redução), apresentando-se como moderadamente tóxico sobre as larvas com exceção de pyridaben 20CE a 20 g.i.a/100 1 com $77 \%$ de redução ou seja, tóxico. Oxido fenbutatin foi o mais seletivo às mesmas a 1 DAA (26\%) sendo moderadamente tóxico (59\%) a 7 DAA. A partir dos 7 DAA a população declinou chegando a 0 individuo/planta, inclusive na testemunha. Como observado para $P$. egena, o declínio do número de larvas resultou em aumento no número de adultos de $C$. citricola.

Outros coccinelideos como C. sanguinea, 
Azya sp. e Calloeneis sp. foram avaliados conjuntamente, ou seja contou-se o número de indivíduos dessas espécies como um complexo (Tabela 2). No entanto, as densidades desses foram extremamente baixas $(0,8$ a 1,8 indivíduos/planta). Também o número de crisopídeos foi baixo (0,3 a 2 indivíduos/planta) tanto em 1993 como em 1994 (Tabela 3), não permitindo uma análise acurada do efeito dos produtos sobre esses inimigos naturais.

Quanto aos ácaros fitoseídeos (Tabela 3), nota-se que a 1 DAA que pyridaben nas diferentes dosagens e formulações foi o mais tóxico (>86\% de redução), seguido de óxido fenbutatin $(76 \%)$ e cyhexatin (67\%). Dong et al. (1992), trabalhando em casa de vegetação, verificaram após liberação de Phytoseiulus persimilis Athias-Henriot em plantas de feijão que em plantas tratadas com pyridaben a redução no número do predador/planta foi maior que $70 \%$. Entre 1 e 14 DAA todos os produtos apresentaram alta toxicidade aos ácaros predadores (> 78\% de redução). Aos 21 DAA uma recuperação dos fitoseídeos foi observada nas parcelas tratadas com óxido fenbutatin (46\% de sobrevivência), o que não ocorreu com pyridaben que apresentou um efeito residual elevado (> 93\% de redução), seguido por cyhexatin $(84 \%)$. Em relação a cyhexatin, os resultados são semelhantes aos de Morse et al. (1987), que obteve, em laboratório mortalidade maior que $80 \%$ em Euseuis stipulatus Athias-Henriot. No entanto, no que se refere a óxido fenbutatin, os autores obtiveram menos que $10 \%$ de mortalidade. Ainda quanto a cyhexatin Bittencourt \& Cruz (1988) estudaram o efeito de defensivos sobre Amblyseius saopauius Denmark \& Muma e Iphiseiodes zuluagai em citros no Rio Grande do Sul e obtiveram mais que $80 \%$ de redução dos fítoseídeos aos 14 dias após a aplicação.

A partir dos resultados obtidos pode se concluir que todos os produtos foram seletivos a adultos de P. egena. Óxido fenbutatin foi seletivo, pyridaben levemente tóxico e cyhexatin moderadamente tóxico às larvas da mesma. Pyridaben $20 \mathrm{CE}$ foi levemente tóxico, pyridaben 75 PM foi moderadamente tóxico, cyhexatin tóxico e óxido fenbutatin seletivo a adultos de C. citricola. Pyridaben $20 \mathrm{CE}$ e 75 PM foram moderadamente tóxicos, cyhexatin tóxico e óxido fenbutatin levemente tóxico a larvas de $C$. citricola. Todos os acaricidas foram tóxicos aos ácaros predadores, entretanto, óxido fenbutatin apresentou menor efeito residual sobre os mesmos.

\section{Literatura Citada}

Bittencourt, M.A. L. \& F. Z. da Cruz. 1988. Toxicidade de produtos químicos sobre ácaros predadores (Acari: Phytoseiidae) em citros. An. Soc. Entomol. Brasil 17: 250-261.

Dong, H. R, V. J. Guo \& J. H. Zhao. 1992. Influence of six commonly used pesticidss at sublethal concentrations on the oviposition, hatching and settlement of Phytoseilus persimilis (Acari; Phytoseiidae). Chin. J. Biol. Contr. 8:106-109.

Gravena, S. 1984. Manejo integrado de pragas dos citros. Laranja 5: 325-361.

Hassan, S. A. 1985. Standard methods to test side-effects of pesticides on natural enemies of insects and mites developed by the IOBC/WRPS Working Group: Pesticides and Beneficial Organisms. Bull. OEPP/EPPO 15:214-255.

Henderson, C. F. \& E. W. Tilton. 1955. Tests with acaricides against the brown wheat mite. J. Econ. Entomol. 63:1536-1539.

Hoyt, C. S. \& E. C. Buris. 1974. Integrated control in fruit pests. Annu. Rev. Entomol. 19:231-235.

Matuo,T. 1990. Métodos de aplicação de de- 
fensivos. In Tecnologia de aplicação de defensivos. Jaboticabal, Funep, 139p.

Moraes, L. A. H. de, J. Braun, O. de Menezes Porto, L. M. Finkler, P. R. Mendes Gimenez, C. C. Camerini \& M. de Oliveira Conte. 1995. Controle químico do ácaro da leprose Brevipalpus phoenicis (Geijskes,, 1939) (Acari, Tenuipalpidae) em citros. Pesq. Agropec. Gaúcha 1:7-11.

\section{Morse , J. G., T. S. Bellows, L. K. Gaston \& Y.} Iwata. 1987. Residual toxicity of acaricides to three beneficial species on California citrus. J. Econ. Entomol. 80:953-960.

Santos, A. C. 1995. Aspectos bioecológicos e seletividade de agroquímicos a Coccidophilus citricola Brèthes (Coleoptera: Coccineilidae). Tese de mestrado, FCAV/ UNESP,Jaboticabal,91p.

Santos, A. C. \& S. Gravena. 1995. Eficiência de diflubenzuron para o ácaro da falsa ferrugem Phyllocoptruta oleivora (Ash.) (Acari: Eriophyidae) e seletividade à Pentilia egena (Muls.) (Coleoptera: Coccineilidae) e ácaros predadores (Acari: Phytoseiidae). An. Soc. Entomol. Brasil 24: 345-351.

Recebido em 21/07/95. Aceito em 12/02/97. 